\title{
Dissolution of the second phase particles in the course of the equal channel angular pressing of diluted $\mathrm{Cu}-\mathrm{Cr}-\mathrm{Zr}$ alloy
}

\author{
I. A. Faizov ${ }^{1,2, \uparrow}$, R. R. Mulyukov ${ }^{3}$, D. A. Aksenov ${ }^{4}$, S. N. Faizova ${ }^{1,3}$, N. V. Zemlyakova ${ }^{5}$, \\ K. R. Cardoso ${ }^{6}$, Yu. Zeng ${ }^{7}$ \\ †iafaiz@mail.ru
}

\author{
${ }^{1}$ Ufa State Petroleum Technological University, 1 Kosmonavtov str., Ufa, 450062, Russia \\ ${ }^{2}$ «BASHNIPINEFT», 86/1 Lenina str., Ufa, 450006, Russia \\ ${ }^{3}$ The Institute for Metals Superplasticity Problems of RAS, 39 Khalturina str., Ufa, 450001, Russia \\ ${ }^{4}$ Institute of Molecule and Crystal Physics of RAS, 71 Prospect Oktyabrya, Ufa, 450000, Russia Россия \\ ${ }^{5}$ Institute of Problems of Mechanical Engineering of RAS, 85 Belinsky str., Nizhny Novgorod, 603024, Russia \\ ${ }^{6}$ Universidade Federal de São Paulo, Rua Talim, 330,Vila Nair, 12231280, São José dos Campos, São Paulo, Brasil \\ ${ }^{7}$ Beijing Aeronautical Manufacturing Technology Research Institute, Ba-li-qiao, P.O. Box 863, Beijing, 100024, China
}

\begin{abstract}
Changes of properties and the second phase particles' distribution in diluted CuCrZr alloy in the course of equal channel angular pressing (ECAP) and post-deformation annealing are investigated. To decrease the concentration of alloying elements dissolved in the copper matrix, samples of the alloy were thermally aged prior to the ECAP. Temperature of annealing, $450^{\circ} \mathrm{C}$, was the same as the one used for the subsequent post-deformation aging, and the time of annealing was long enough to reach a practically equilibrium state. The enthalpy release rate curve of the ECAP-processed sample, unlike the one for the initial state exhibits an exopeak in the temperature range $440 . .470^{\circ} \mathrm{C}$, which is typical for breakdown of the solid solution in alloys of this system. The post-deformation aging causes a significant increase in the tensile strength as compared to the value immediately after the ECAP. This increase can be related only to the dispersion strengthening that is confirmed by the observation of higher density of particles after the aging. These facts unambiguously indicate that the solid solution at the aging temperature is supersaturated, i.e. its concentration increased in the course of the ECAP due to the deformation-induced dissolution of the second phases. This conclusion agrees with the behavior of the electrical conductivity that noticeably decreases upon the ECAP and recovers its initial value after the aging. As the density of structural defects is significantly different in the initial and final states, the observed decrease can be related only to the lattice distortion due to dissolved foreign atoms.
\end{abstract}

Keywords: deformation-induced dissolution, second phase particles, copper alloy, equal channel angular pressing, severe plastic deformation, phase transitions.

УДК 544.22

\section{Растворение частиц вторых фаз в низколегированном медном сплаве системы Cu-Cr-Zr при обработке методом равноканального углового прессования}

\author{
Фаизов И. А. ${ }^{1,2, \dagger}$, Мулюков Р. Р. ${ }^{3}$, Аксенов Д. А. ${ }^{4}$, Фаизова С. Н. ${ }^{1,3}$, Землякова Н. В. ${ }^{5}$, \\ Cardoso K. R. ${ }^{6}$, Zeng Yu. ${ }^{7}$ \\ ${ }^{1}$ Уфимский государственный нефтяной технический университет, ул. Космонавтов, 1, Уфа, 450062, Россия \\ ${ }^{2} \mathrm{OOО}$ «БашНИПИнефть», ул. Ленина, 86/1, Уфа, 450006, Россия \\ ${ }^{3}$ Институт проблем сверхпластичности металлов РАН ул. Халтурина 39, Уфа, 450001, Россия \\ ${ }^{4}$ Институт физики молекул и кристаллов УНЦ РАН, Пр.Октября, 71, Уфа, 450000, Россия \\ ${ }_{5}^{5}$ Институт проблем машиностроения РАН, ул. Белинского, 85, Нижний Новгород, 603024, Россия \\ ${ }^{6}$ Universidade Federal de São Paulo, Rua Talim, 330,Vila Nair, 12231280, São José dos Campos, São Paulo, Brasil \\ ${ }^{7}$ Beijing Aeronautical Manufacturing Technology Research Institute, Ba-li-qiao, P.O. Box 863, Beijing, 100024, China
}

В работе исследуется изменение физико-механических свойств и эволюция распределения частиц вторых фаз в низколегированном сплаве системы $\mathrm{Cu}-\mathrm{Cr}-\mathrm{Zr}$ в процессе обработки методом равноканального углового прессования 
(РКУП) и пост-деформационного отжига. Для снижения концентрации твердого раствора легирующих элементов в медной матрице перед деформированием образцы сплава были подвергнуты термическому старению. Температура отжига, $450^{\circ} \mathrm{C}$, была той же самой, что использовалась затем для пост-деформационного старения, а время выдержки выбрано достаточным для приближения к практически равновесному состоянию. График скорости выделения энтальпии у образцов, подвергнутых РКУП, в сравнении с исходным состоянием обнаруживает экзопик в области температур $440 . .470^{\circ} \mathrm{C}$, характерных для распада пересыщенного твердого раствора в данном типе сплавов. Пост-деформационный отжиг приводит к заметному увеличению предела прочности по сравнению со значением для образцов после РКУП, которое можно связать только с дисперсионным упрочнением, что подтверждается непосредственным наблюдением возрастания плотности частиц. Эти два факта однозначно указывают на то, что твердый раствор является пересыщенным при температуре старения, то есть, что его концентрации в ходе РКУП возросла вследствие деформационно-индуцированного растворения частиц вторых фаз. Сделанный вывод согласуется с изменениями электропроводности сплава, заметно снижающейся в ходе деформирования и восстанавливающей исходное значение после старения. Так как плотность структурных дефектов в конечном и исходном состояниях материала существенно отличается, наблюдаемое уменьшение проводимости может быть связано только с искажениями решетки из-за растворенных атомов.

Ключевые слова: деформационно-индуцированное растворение частиц вторых фаз; медный сплав, равноканальное угловое прессование, интенсивная пластическая деформация, фазовые превращения.

\section{1. Введение}

В настоящее время накоплен большой экспериментальный материал об аномальных фазовых превращениях, сопровождающих интенсивную пластическую деформацию (ИПД) металлических сплавов [1-13]. В частности, явление деформационно-индуцированного растворения частиц вторых фаз подробно изучено в сплавах на основе железа [11-13]. При холодной ИПД легирующие элементы, такие как C, N, Ni переходят из частиц вторых фаз в твердый раствор, причем его результирующая концентрация может стать выше насыщенной концентрации. Несмотря на большое количество экспериментальных данных, единого мнения относительно механизмов деформационно-индуцированного растворения не существует. В [11-13] предлагается схема, согласно которой на начальной стадии ИПД происходит, главным образом, измельчение частиц и накопление в них структурных дефектов, а на более развитой стадии с увеличением вклада “некристаллографических" механизмов деформации, сопровождающихся интенсивной генерацией вакансий, становится более заметным отрыв атомов от частиц и их дрейф в поле движущихся дислокаций. В работах [14-15] развивается подход, основанный на термодинамическом описании неравновесных квазистационарных состояний при ИПД, учитывающем высокую динамическую плотность структурных дефектов. Включение в термодинамические потенциалы энергий адсорбции примесных атомов на дислокациях и границах фрагментов приводит к сдвигу границ сосуществования фаз, что может объяснять растворение частиц при деформировании.

В данной работе изучается деформационно-индуцированное растворение легирующих элементов в меди при РКУП сплава системы Cu-Cr-Zr. Большинство результатов по деформационно-индуцированному растворению получено с использованием наиболее «жестких» методов ИПД (кручение на наковальнях Бриджмена, механическое сплавление и др.) и в системах, имеющих заметную равновесную концентрацию твердого раствора при температуре эксперимента, либо с элементами, образующими ковалентную связь с атомами матрицы. РКУП дает относительно более низкую накопленную деформацию, а равновесная растворимость хрома в меди при температурах близких к комнатной пренебрежимо мала. Это делает данный случай заметно отличающимся от изученных к настоящему времени, и его анализ представляет интерес для понимания механизмов деформационно-индуцированного растворения.

Как уже отмечалось, наиболее изученным классом материалов являются сплавы на основе железа, у которых ряд физических характеристик, в частности, температура Кюри [11-13], чувствительны к концентрации растворенных легирующих элементов, что делает такие сплавы удобным объектом исследования. Для низколегированных хромовых бронз определение концентрации твердого раствора представляет собой сложную задачу, для решения которой используются косвенные подходы: измерения электропроводности, микротвердости и термический анализ, включая дифференциальную сканирующую калориметрию (ДСК). Важным источником информации о фазовых превращениях являются наблюдения за изменениями характеристик ансамбля частиц вторых фаз. Когда в системе присутствует пересыщенный твердый раствор, эти изменения потенциально зависят от трех различных процессов: механическое измельчение - дислокационное перерезание либо хрупкое разрушение частиц, зарождение и рост частиц за счет деформационно-стимулированного распада твердого раствора и деформационно-индуцированное растворение. Относительный вклад этих процессов можно варьировать за счет специально подготовленных исходных состояний сплава. Исследования на предварительно состаренном сплаве, имеющем минимальную концентрацию растворенных в матрице легирующих элементов, позволят исключить, по крайней мере на начальных этапах деформирования, влияние деформационно-стимулированного распада и наиболее полно выделить эффект растворения. 


\section{2. Материал и методики эксперимента}

Для получения исходного состояния образцы дисперсионно-упрочняемого сплава $\mathrm{Cu}-0,6 \% \mathrm{Cr}-0,1 \% \mathrm{Zr}$ выдерживали при температуре $450^{\circ} \mathrm{C}$ в течение 4 ч. Температура была выбрана с целью обеспечения распада ТР в соответствии с данными калориметрических исследований сплава аналогичного состава, приведенными в работе [16]. Величина коэффициента решеточной диффузии хрома [17] в меди при данной температуре достаточна для того, чтобы достичь равновесной концентрации твердого раствора за несколько часов. В соответствии с диаграммой состояния [18], эта концентрация является настолько низкой, что позволяет пренебречь эффектом деформационно-стимулированного распада твердого раствора, по крайней мере на начальных этапах РКУП. Критерием достижения равновесного состояния являлась стабилизация значений электропроводности и микротвердости. На контрольных образцах сплава была проведена также закалка в воду с температуры $1050^{\circ} \mathrm{C}$. Для закаленных образцов, образцов в исходном состоянии для РКУП и после второго прохода РКУП на дифференциальном сканирующем калориметре NETZSCH DSC 204F1 Phoenix были получены графики скорости изменения энтальпии при линейном нагреве $40^{\circ} \mathrm{C} /$ мин до $550^{\circ} \mathrm{C}$.

Образцы для РКУП имели диаметр 10 мм и длину 60 мм. РКУП проводили на установке с углом пересечения каналов $90^{\circ}$ по маршруту Вс при температуре $150^{\circ} \mathrm{C}$, количество проходов от 1 до 8 с исследованием микроструктуры и свойств после каждого прохода. Пост-деформационное старение (ТО) проводили при той же, что и предварительная термообработка, температуре, $450^{\circ} \mathrm{C}$, в течении 30 мин. Микроструктуру образцов после каждого этапа обработки исследовали методами световой, растровой (РЭМ) и просвечивающей (ПЭМ) электронной микроскопии на приборах JEOL JSM-6390 и JEOL-2100, соответственно.

Количественный анализ размеров и характера распределения частиц и их идентификацию на микроуровне проводили на репликах по методикам, описанным в работе [19]. В исследуемом материале наблюдаются частицы, имеющие размеры от нанометров до нескольких микрон, причем плотность их распределения в целом уменьшается с ростом размера. Вследствие этого, для определения характеристик распределения требуется проводить измерения на двух масштабных уровнях: на мезоуровне (РЭМ) и микроуровне (ПЭМ). На мезоуровне минимальный размер наблюдаемых частиц ограничен разрешением и составляет $300 . .400$ нм. На микроуровне для подсчета использовались составленные из нескольких микрофотографий панорамы, имеющие линейные размеры поля зрения $2 . .3$ мкм и содержащие одну-две тысячи частиц. Увеличение размера панорам нецелесообразно ввиду большой трудо емкости подсчета, а данное количество частиц достаточно для получения достоверной статистики распределения.

Измерения микротвердости и испытания на растяжение проводили по стандартным методикам [20] на микрометре Micromet-5101 и установке INSTRON-1114. Электропроводность измеряли вихретоковым методом на приборе ВЭ-27НЦ/6 согласно ГОСТ 27333-87.

\section{3. Результаты и обсуждение}

В ходе РКУП происходит быстрое измельчение структуры сплава. Уже после двух проходов средний размер фрагментов составляет примерно 120 нм и мало меняется с дальнейшим накоплением деформации. После пост-деформационного отжига этот размер находится в интервале $120 . .150$ нм, т. е. структура образцов остается ультрамелкозернистой.

На Рис. 1 приведены кривые скорости изменения энтальпии для трех образцов. Кривая для образца после двух проходов РКУП в сравнении с исходным состоянием демонстрирует экзопик в области $450 . .470^{\circ} \mathrm{C}$ с выделением энтальпии, приблизительно равным 140 Дж/кг. Его положение практически совпадает с положением выраженного экзопика с энтальпией 956 Дж/кг на кривой для закаленного образца, который однозначно связан с распадом твердого раствора, поскольку для крупнозернистого отожженного материала эффекты дислокационного возврата, рекристаллизации и других возможных экзотермических процессов перестройки структуры пренебрежимо малы. Также по данным [21], пики на ДСК кривых закаленных образцов сплавов близкого состава, подвергнутых РКУП, в данной температурной области соответствуют реакциям распада твердого раствора с образованием различных медь-хромовых и медь-хромциркониевых интерметаллидов. Таким образом, появление данного экзопика на ДСК кривой образца после РКУП, с учетом факта, что после даже более длительного нагрева до температуры $450^{\circ} \mathrm{C}$ образец сохраняет УМЗ структуру, указывает на повышение концентрации ТР по сравнению с исходной, источником которого может быть только частичное растворение вторых фаз.

Для микроуровня эволюция ансамбля частиц вторых фаз в ходе эксперимента иллюстрируется Табл. 1. Во всех

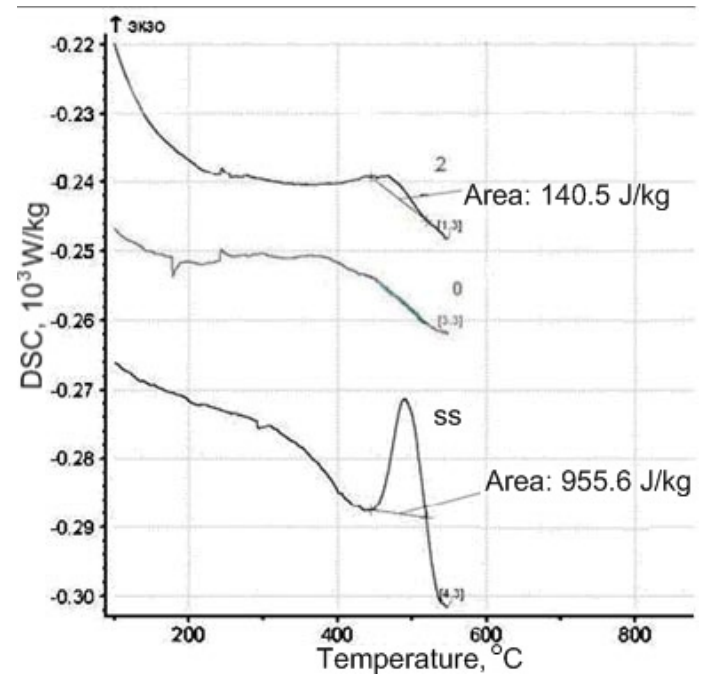

Рис. 1. Скорость выделения энтальпии при линейном нагреве: TP - контрольный закаленный образец с пересыщенным твердым раствором. 0 - исходное состояние перед РКУП; 2 - после двух циклов РКУП.

Fig. 1. The enthalpy release rate under linear heating: SS - quenched samples with supersaturated solid solution. 0 - sample in the initial state before ECAP; 2 - sample after 2 ECAP passes. 
трех состояниях плотность распределения частиц уменьшается с ростом их размера. РКУП приводит к сильному снижению плотности частиц размером > 20 нм по сравнению с исходным состоянием - среднее расстояние между такими частицами становится больше размера поля наблюдения. В тоже время плотность частиц с размерами $<10$ нм увеличивается приблизительно в 5 раз. Таким образом, можно констатировать, что РКУП вызывает измельчение частиц.

Одним из предположительных механизмов процесса измельчения частиц может быть хрупкое разрушение под действием напряжений от заторможенных частицами дислокационных скоплений. При этом внутри частиц могут присутствовать незавершенные сдвиги, создающие дополнительное внутреннее напряжение. Кроме того, ступеньки на границе частица-матрица, образующиеся при прохождении дислокаций, действуют как концентраторы напряжения. С уменьшением размера частиц облегчается их огибание дислокациями, вследствие чего уменьшается размер дислокационных скоплений и, соответственно, действующее внутри частиц напряжение, поэтому следует ожидать существования некоторого критического размера частиц, ниже которого разрушение по данному механизму становится невозможным. По приведенным данным, этот размер равен 10..20 нм. В рамках данного предположения плотность частиц в диапазоне < 10 нм возрастает за счет осколков более крупных. Уменьшение среднего размера частиц со смещением наиболее вероятного размера в сторону наиболее мелких наблюдается также и на мезоуровне (Рис. 2). Следует отметить, что для этого масштабного уровня измельчение частиц не может сводиться лишь к растворению и имеет, следовательно, механическую природу.

Фрагменты частиц после разрушения имеют ребра, т.е. участки с очень высокой кривизной поверхности. Согласно уравнению Гиббса-Томсона в их окрестности локальная равновесная концентрация твердого раствора значительно превышает объемную, что и делает возможным частичное растворение.

Пост-деформационный отжиг увеличивает на микроуровне плотность частиц в диапазонах размеров до $70 . .80$ нм. Для частиц этого размера среднее расстояние между частицами уменьшается до критического

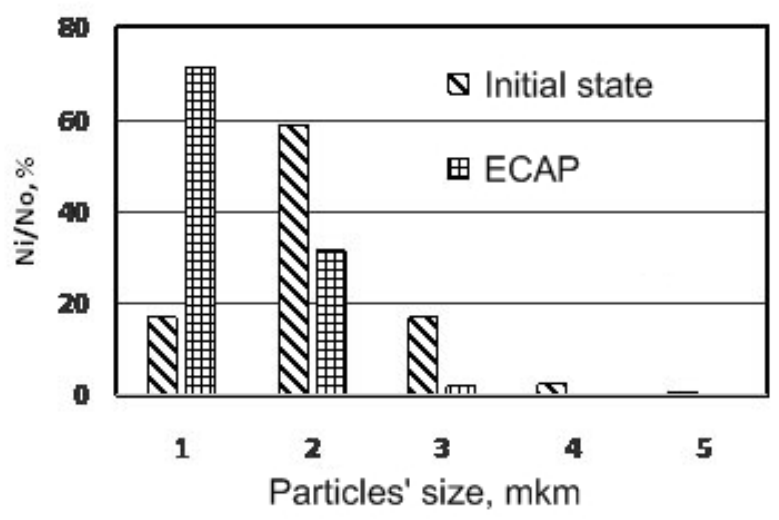

Рис. 2. Распределение частиц по размерам на мезоуровне (РЭМ) в исходном состоянии и после 8 проходов РКУП.

Fig. 2. Particles' size distribution on the mesoscale (SEM) in the initial state and after 8 passes of ECAP.

для данного метода измерения значения 2..3 мкм линейных размеров поля наблюдения микропанорамы. Более крупные частицы не наблюдаются из-за того, что среднее расстояние между ними остается больше этой величины. Плотность частиц в диапазоне $<10$ нм увеличивается в два раза, среднее расстояние между ними уменьшается до 40 нм.

Увеличение плотности частиц при пост-деформационном отжиге после 8 проходов РКУП служит подтверждением образования в условиях РКУП пересыщенного твердого раствора, поскольку в исходом состоянии его концентрация в образцах была близка к равновесной для температуры старения. Средний диффузионный путь атомов хрома, оцениваемый как $\sqrt{D t}$, где $D-$ коэффициент диффузии [17], при температуре $450^{\circ} \mathrm{C}$ (723 K) и времени отжига $t=30$ мин равен $15 . .30 \mathrm{Hм}$, что хорошо согласуется с наблюдаемой плотностью частиц. В работе [22] показано, что в низколегированном сплаве $\mathrm{Cu}-\mathrm{Cr}$ распад твердого раствора происходит через последовательное образование интерметаллидов разной степени метастабильности. При этом, в первую очередь, благодаря низкой поверхностной энергии, образуются обогащенные медью частицы с ГЦК решеткой, что объясняет быстрое зарождение большого количества частиц при пост-деформационной термообработке.

Табл. 1. Изменение плотности распределения частиц в зависимости от размера на разных этапах обработки сплава (ПЭМ). Table 1. Changes in the distribution density of particles depending versus their sizes on different stages of processing (TEM).

\begin{tabular}{|c|c|c|c|}
\hline \multirow{2}{*}{$\begin{array}{c}\text { Интервал размеров, нм } \\
\text { Size range, nm }\end{array}$} & \multicolumn{3}{|c|}{$\begin{array}{c}\text { Плотность частиц, мкм } \\
\text { Distribution density, } \text { mm }^{-2}\end{array}$} \\
\cline { 2 - 4 } & $\begin{array}{c}\text { Исходное } \\
\text { Initial }\end{array}$ & $\begin{array}{c}\text { PКУП 8 пр } \\
\text { ЕСАР 8 ps }\end{array}$ & $\begin{array}{c}\text { Пост-деформационное старение } \\
\text { Post-deformation aging }\end{array}$ \\
\hline$<10$ & 58 & 303 & 610 \\
\hline $10 . .20$ & 9 & 9 & 84 \\
\hline 20.30 & 5 & 0 & 18 \\
\hline $30 . .40$ & 0 & 0 & 9 \\
\hline $40 . .50$ & 0 & 0 & 3 \\
\hline $50 . .60$ & 0 & 0 & 1 \\
\hline $60 . .70$ & 0 & 0 & 1 \\
\hline $70 . .80$ & 0 & 0 & 4 \\
\hline
\end{tabular}


Электропроводность сплава в исходном состоянии составляет 84\% IACS. РКУП приводит к ее снижению до 78\%, а пост-деформационная термообработка восстанавливает исходное значение. Электросопротивление медной матрицы при комнатной температуре малочувствительно к дефектам масштаба дислокаций и границ зерен и зависит, в основном, от искажений решетки на масштабах порядка межатомных расстояний. Это связано с малой длиной волны электрона при такой температуре и подтверждается экспериментом на технически чистой меди, электропроводность которой при аналогичной обработке остается постоянной в пределах ошибки. Повышение концентрации растворенных в медной матрице атомов увеличивает искажения решетки и объясняет снижение электропроводности сплава после РКУП.

Предел прочности сплава в исходном состоянии равен 560 МПа, после РКУП он возрастает до $655 \mathrm{MПа,}$ а пост-деформационное старение приводит к его дальнейшему увеличению до 705 МПа. На этапе РКУП происходит сильное измельчение структуры сплава и увеличивается плотность дислокаций. Увеличение вклада зернограничного и дислокационного упрочнения способно, в принципе, объяснить сопутствующее возрастание прочности. При пост-деформационном отжиге происходит частичный дислокационный возврат и, как было уже сказано, некоторое огрубление УМЗ структуры, поэтому единственным объяснением возрастания прочности является увеличение вклада дисперсионного упрочнения, что согласуется с наблюдаемым увеличением плотности частиц.

\section{4. Выводы}

При РКУП образцов сплава $\mathrm{Cu}-\mathrm{Cr}-\mathrm{Zr}$, предварительно подвергнутых старению, происходит повышение концентрации твердого раствора легирующих элементов в меди.

РКУП вызывает также изменения в распределении частиц по размерам, которые, по-видимому, является результатом как их механического измельчения. Наблюдение механического измельчения частиц в ходе ИПД, приводящей одновременно к увеличению концентрации твердого раствора, позволяет выдвинуть предположение о взаимосвязи этих процессов.

\section{Литература/References}

1. A. Ye. Ermakov. Phys. Metals Metallogr. 11, 4 (1991) (in Russian) [А.Е. Ермаков, ФММ, 11, 4 (1991)]

2. P.H. Shingu, K. N. Ishihara, J. Kuyama. Proc. of ThirtyFourth Japan Congress on Mat. Res., Kyoto, Japan, 19 (1991).

3. A. Ye. Yermakov. Mat. Sci. Forum, 179 - 181, 455 (1995).

4. R. B. Schwarz, W.L. Johnson. Phys. Rev. Lett. 51, 415 (1983). DOI: 10.1103/PhysRevLett.51.415

5. C.E. Rodriquez Torres, F. N. Sanches, L.A. Mendoza Zeilis. Phys. Rev. B51 (18), 12142 (1995). DOI: 10.1103/ PhysRevB.51.12142

6. U. Czubayko, N. Wanderka, V. Naundorf, V. A. Ivchenko,
A. Ye. Yermakov, M. A. Uimin, H. Wollenberger. Mater. Sci and Eng. A327, 54 (2002). DOI: 10.1016/S09215093(01)01875-5

7. N. Wanderka, U. Czubayko, V. Naundorf, V. A. Ivchenko, A. Ye. Yermakov, M.A. Uimin, H. Wollenberg. Ultramicroscopy, 89, 189 (2001). DOI: 10.1016/S03043991(01)00104-8

8. C. Bansal, Z. Q. Gao, L. B. Hong, B. Fultz. J. Appl. Phys. 76, 5961 (1994). DOI: 10.1063/1.358375

9. S.A. Starikov, A.R. Kuznetsov, Yu.N. Gornostyrev, V.V. Sagaradze. Diagnostics, Resource and Mechanics of materials and structures. 6, 48 (2016). DOI: 10.17804/2410-9908.2016.6.048-062

10. A.V. Korolev, E.G. Gerasimov, V.A. Kazantsev, A. I. Deryagin, V. A. Zavalishin. Phys. Metals Metallogr. 79, 136 (1995). (in Russian). [А. В. Королев, Е. Г. Герасимов, В. А. Казанцев, А. И. Дерягин, В. А. Завалишин. ФММ. 79 (2), 136 (1995)].

11. V.V. Sagaradze, S.V. Morozov, V.A. Shabashov, L.N. Romashev, V.N. Kuznetsov// Phys. Metals Metallogr. 66, 328 (1988) (in Russian). [В. В. Сагарадзе, С. В. Морозов, В.А. Шабашов, Л.Н. Ромашев, Р. И. Кузнецов. ФММ. 66 (2), 328 (1988).]

12. V.V. Sagaradze, V.A. Shabashov, T.M. Lapina, N. L. Pecherkina, V. P. Pilyugin. The Phys. Metals Metallogr. 78 (6), 619 (1994) (in Russian). [В.В. Сагарадзе, В.А. Шабашов, Т.М. Лапина, Н.Л. Печеркина, В. П. Пилюгин. ФММ. 78 (6), 49 (1994).]

13. V. V. Sagaradze, V.A. Shabashov. Phys. Metals Metallogr. 112 (2), 146 (2011) (in Russian). [В.В. Сагарадзе, B. А. Шабашов. ФММ. 112 (2), 155 (2011)].

14. O.I. Gorbatova, Yu.N. Gornostyrev, P.A. Korzhavyi and A. V. Ruban. Phys. Metals Metallogr. 117 (13), 1293 (2016).

15. I. K. Razumov, Yu. N. Gornostyrev and M. I. Katsnelson. Phys. Metals Metallogr. 118 (4), 362 (2017).

16. A. Vinogradov, V. Patlan, Y. Suzuki, K. Kitagawa, V.I. Kopylov. Acta Mater. 50, 1639 (2002). DOI: 10.1016/ S1359-6454(01)00437-2

17. A. Almazouzi, M.-P. Macht, V. Naundorf, G. Neumann. Phys. stat. sol. (a). 167, 15 (1998). DOI: 10.1002/ (SICI)1521-396X(199805)167:1<15::AID-PSSA15>3.0. $\mathrm{CO} ; 2-8$

18. D. J. Chakrabarti, D. E. Laughlin. The Cr-Cu (ChromiumCopper) System Bulletin of Alloy Phase Diagram. 5 (1),59 (1984). DOI: 10.1007/BF02868543

19. S. N. Faizova, G.I. Raab, N.G. Zaripov, D.A. Alsenov, I. A. Faizov. Phys. Mezomech. 18 (4), 87 (2015) (in Russian). [С.Н. Фаизова, Г.И. Рааб, Н.Г. Зарипов, Д. А. Аксенов, И. А. Фаизов. Физическая мезомеханика. 18 (4), 87 (2015).]

20. V.S. Zolotarevsky. Mechanical properties of metals. M. MISIS (1998) 400 p. (in Russian). [В.С. Золоторевский. Механические свойства металлов. М. МИСИС (1998) 400 с.]

21. A. Vinogradov, Y. Suzuki, T. Ishida, K. Kitagawa and V.I. Kopylov. Mater Transactions. 45 (7), 2187 (2004). DOI: $10.2320 /$ matertrans.45.2187

22. A. Chbihi, X. Sauvage, D. Blavette. Acta Mat. 60 (11), 4575 (2012). DOI: 10.1016/j.actamat.2012.01.038 